\title{
Cutaneous squamous carcinoma in a patient with diabetic foot: an unusual evolution of a frequent complication
}

\author{
Alessandro Mantovani', Ilaria Teobaldi', Vincenzo Stoico', Fabrizia Perrone1, \\ Marina Zannoni², Luca Cima², Massimiliano Bruti³, Lucia Mingolla', \\ Maddalena Trombetta' and Enzo Bonora' \\ 1Division of Endocrinology, Diabetes and Metabolism, Department of Medicine, 2Division of Pathology Unit, \\ Department of Diagnostics and Public Health, and 'Division of Plastic Surgery, Department of Surgery, University and \\ Azienda Ospedaliera Universitaria Integrata of Verona, Verona, Italy
}

Correspondence should be addressed to A Mantovani

Email

alessandro.mantovani@ univr.it

\section{Summary}

After basal cell carcinoma, the cutaneous squamous cell carcinoma ( $\mathrm{SCC}$ ) is the second most frequent non-melanoma skin cancer worldwide, and, classically, arises from the upper coats of the epidermis of sun-exposed areas or from skin areas constantly exposed to a chronic inflammatory stimulus. The occurrence of CSCC seems to be linked to several factors, including exposure to sunlight (or other ultraviolet radiations), immunosuppression, chronic scarring conditions and some familial cancer syndromes. Although the majority of CSCCs are adequately eradicated by surgical excision, a subgroup of CSCC may be linked with an increased risk of recurrence, metastasis and death. The incidence of type 2 diabetes mellitus is constantly increasing worldwide. Importantly, diabetes mellitus is a strong risk factor for cancers (including cutaneous tumors) and is highly related with poor cancer outcomes. At present, in the literature, squamous cell carcinoma developing in association with diabetic foot ulcers has been already reported in some reports; however, additional data are needed to make the clinicians aware of this rare, although possible, complication. Therefore, we herein report an unusual case of an elderly man with T2DM and a positive oncological history, presenting a CSCC involving the skin overlying the first toe of left foot. The growing CSCC appeared approximately 3 years after the appearance of a diabetic ulcer.

\section{Learning points:}

- Diabetic foot ulcers are an important and severe complication of diabetes mellitus and often can result in foot amputation.

- Chronic and non-healing diabetic foot ulcers are often observed in clinical practice.

- Clinicians should always take into consideration the malignant degeneration (e.g., cutaneous squamous cell carcinoma) of any chronic non-healing diabetic foot ulcer in elderly T2DM individuals.

- Timely surgical resection of a chronic, non-healing diabetic foot ulcer might preclude the development of a cutaneous squamous cell carcinoma.

\section{Introduction}

The cutaneous squamous cell carcinoma (cSCC) is estimated to be the second most frequent non-melanoma skin cancer worldwide (after basal cell carcinoma), as it represents $20-50 \%$ of all skin cancers $(1,2)$. Traditionally, cSCC appears from the higher coats of the epidermis of sun-exposed areas or from skin areas steadily exposed 
to a chronic inflammatory stimulus $(3,4)$. Cutaneous squamous cell carcinoma often affects middle-aged or elderly individuals and males seem to be more affected than females $(1,2)$. Of note, although the most cases of cSCCs are adequately eradicated by surgical excision, a subgroup of cSCC could be associated with recurrence, metastasis and even death (3). The occurrence of cSCC is related to multiple factors, such as exposure to sunlight or other ultraviolet radiations, immunosuppression, human papillomavirus, chronic scarring conditions and some familial cancer syndromes (4).

To date, the total prevalence of type 2 diabetes (T2DM) is estimated to be nearly $8-9 \%$ of the adult population (approximately 420 million individuals) (5). Moreover, it is calculated that the overall prevalence of diabetes mellitus will rise to roughly 700 million in 2035 and that approximately $75-80 \%$ of these individuals will live in developing countries (5). In addition to the increased risk of vascular and infectious disease (owing to T2DM), recent data strongly suggest that patients with T2DM have a higher risk of dying from cancer, including cutaneous tumors, compared to those without T2DM (6).

To date, in the literature, cSCC developing in association with a diabetic foot ulcer has been already described in some reports $(7,8,9)$, but more information is required in order to make the clinicians aware of this rare (albeit possible) complication.

Therefore, we report herein an unusual case of an elderly man with T2DM and prior history of colon adenocarcinoma, presenting a CSCC involving the skin overlying the first toe of left foot. The growing cSCC occurred nearly 3 years after the appearance of a diabetic foot ulcer.

\section{Case presentation}

An 83-year-old Caucasian male patient with type 2 diabetes (T2DM) came to our Diabetic Foot Clinic for the appearance of a traumatic ulcer at the apex of the first toe of his left foot in 2015 (Fig. 1, Panel A). The diagnosis of T2DM was made roughly 17 years ago and was treated with metformin with a good glycemic compensation. In addition to T2DM, his medical history included: hypertension, diabetic peripheral neuropathy and a prior history of colon adenocarcinoma eradicated by surgical excision followed by a treatment with capecitabine. A family history of cancer was not reported by the patient. As described previously, the ulcer at the apex of the first toe of left foot arose following a traumatic event (the patient said he had slammed into a bedroom furniture). At the
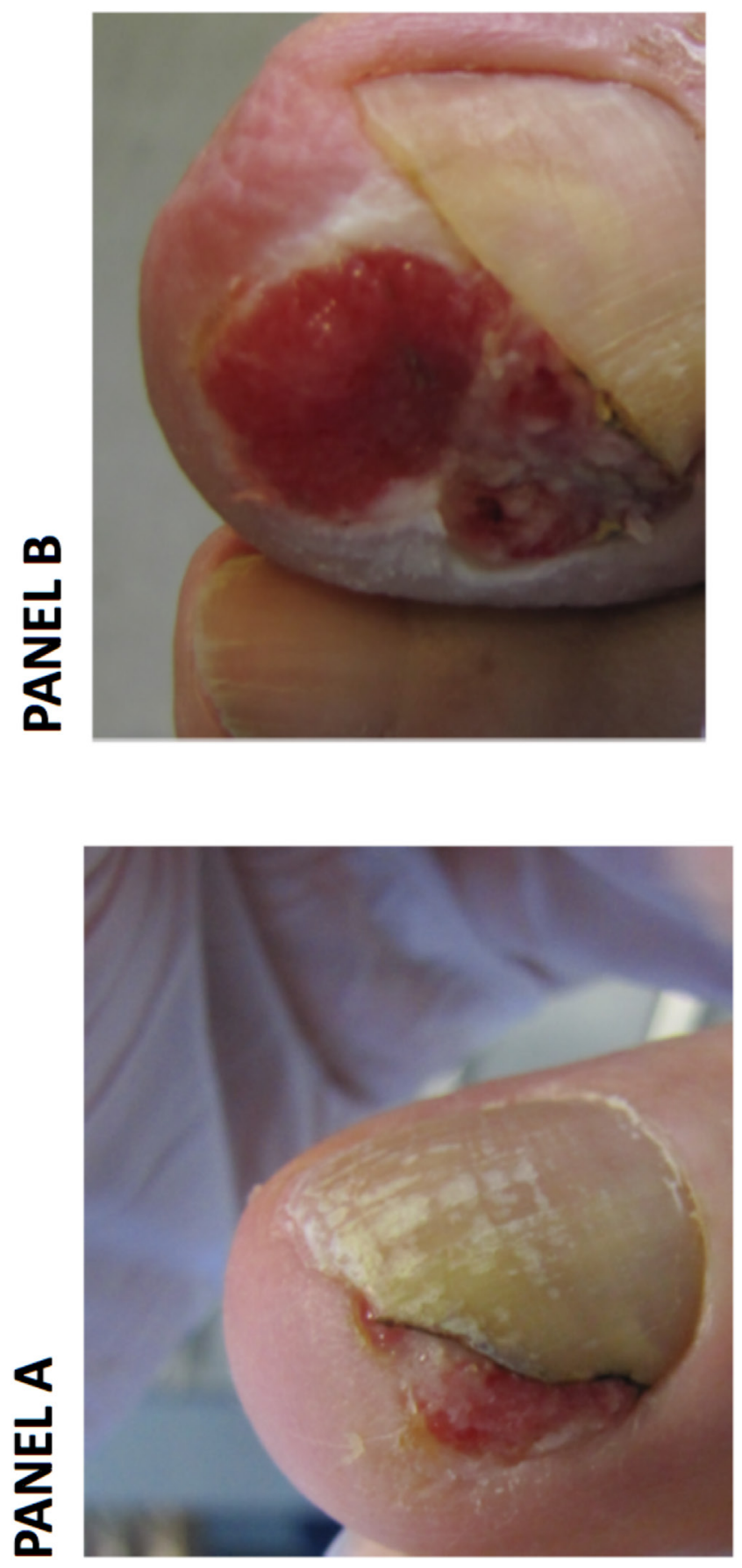

Figure 1

(Panel A) Initial presentation of the traumatic lesion located at the apex of the first toe of the left foot, in the subungual region beneath the toenail. (Panel B) Appearance of a red lobular lesion characterized by a friable consistency at the apex of the first toe (in the subungual region beneath the toenail) of the left foot appeared several years later.

beginning, the ulcer had been treated with a conservative approach by dressings with topical antiseptic and oral antibiotic therapy with amoxicillin-clavulanate, based on Staphylococcus aureus isolated by biopsy performed 
in an ambulatory setting. The blood tests showed white blood cells (WBC): $8.6 \times 10^{9} / \mathrm{L}$ (normal range: 4.3-10), hemoglobin 124 g/L (normal range: 135-170), platelets: $331 \times 100,000 / \mathrm{mm}^{3}$ (normal range: $150-450$ ), fasting plasma glucose: $6.9 \mathrm{mmol} / \mathrm{L}$ (normal range: 3.5-5.5), A1c: $47 \mathrm{mmol} / \mathrm{mol}$ (normal range: <42), alanine aminotransferase (ALT): 20 U/L (normal range: 6-50), gamma-glutamyl transpeptidase (GGT): 16U/L (normal range: 4-60), creatinine: $73 \mu \mathrm{mol} / \mathrm{L}$ (normal range: 53-115) and C-reactive protein (CRP): $8 \mathrm{mg} / \mathrm{L}$ (normal range: $<5)$.

After 5 weeks, the ulcer completely healed. However, 6 months later, the patient returned to our attention for the appearance of a new ulcer in the same area of the lesion described previously. The new lesion appeared pinkish, lobulated with a high tendency to bleeding. Apparently, there had not been any recent trauma that could explain such recurrence, but it is important to highlight that our patient had diabetic peripheral neuropathy, a condition that could conceal recurrent trauma owing to unsuitable footwear. Given the unusual characteristics of this lesion, we decided to perform a biopsy, which documented the presence of a pyogenic granuloma. As known, conservative surgical excision is the established choice of treatment for pyogenic granuloma and, therefore, we proposed to our patient a surgical excision, which he categorically refused. Given the unshakable decision of the patient, the subsequent management of the lesion was essentially made by local dressings (i.e., antiseptic and silver nitrate) (10) and use of specific shoes in order to offload the lesion. The patient was constantly informed regarding the need to perform a surgical excision, but he always refused. The conservative treatment (with dressings and offloading systems) has been going on for approximately 18 months; a period wherein we observed a reduction of the size of the lesion followed by frequent recurrences. However, for the next 10 months, the patient followed no longer regular checks at our clinic, until he returned for the appearance of a red lobular lesion with a friable consistency at the apex of the first toe of the left foot, in the subungual region beneath the toenail (Fig. 1, Panel B). Again, the patient denied a recent trauma. The novel unusual appearance of the lesion as well as the detection of small calcifications on radiography (Fig. 2, Panel A) imposed the execution of a new incisional biopsy with subsequent histological examination, which revealed an infiltrate of monomorphic large cells in the dermis with prominent nucleoli and frequent mitoses, but without any vascular or perineural invasion. Immunohistochemical analysis showed a diffuse expression of CK5 and P40
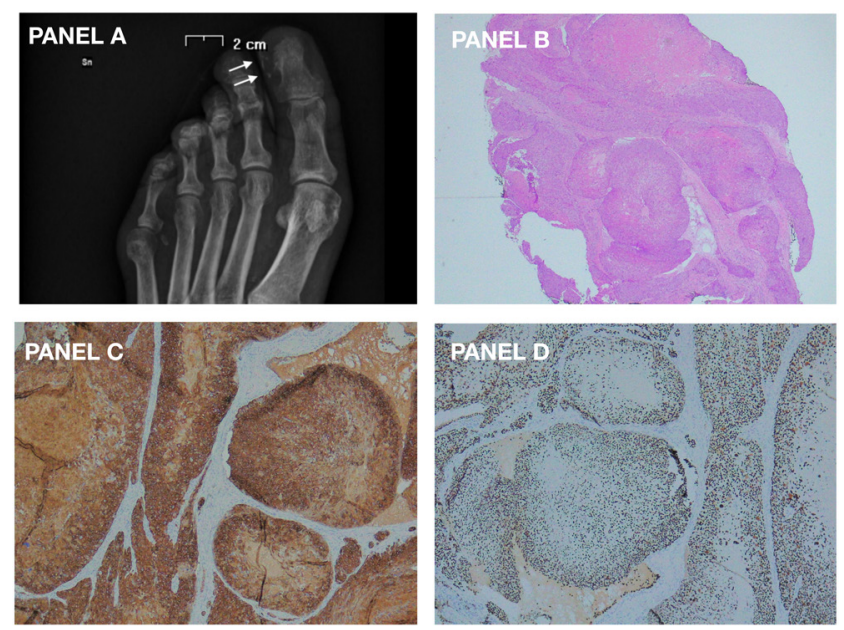

\section{Figure 2}

(Panel A) Small calcifications (white arrows) detected by radiography at the level of the first toe of the left foot. (Panel B) Histological findings of in situ cutaneous squamous cell carcinoma. (Panel C)

Immunohistochemical analysis of CK5. (Panel D) Immunohistochemical analysis of $p 40$

(Fig. 2, Panel B, C and D). The final diagnosis was an in situ cutaneous squamous cell carcinoma.

\section{Treatment, outcome and follow-up}

The clinical case was extensively discussed with oncologist and plastic surgeon who suggested the complete amputation of the toe. The ultrasound of the inguinal and the popliteal cavity did not reveal the presence of suspicious lymph nodes. Therefore, no further radiological examinations were performed.

\section{Discussion}

The cSCC is the one of the most common non-melanoma skin cancer worldwide and, typically, occurs from the upper layers of the epidermis of areas steadily exposed to a chronic inflammatory stimulus $(1,2)$. Importantly, although the majority cases of cSCCs are sufficiently eradicated through the surgical excision, a subset of cSCCs is linked to recurrence, metastasis and even death (3).

Classically, cSCC are examined by clinical examination and skin biopsy for histology (3). The biopsies can be excisional, where the entire lesion is removed, or incisional, in which a part of the lesion is excised. A biopsy provides a histopathological diagnosis. Subsequent investigations can be performed in some cases to assess extent of the disease and often include computed tomography imaging (in order to search for bone and soft tissue expansion) or MRI (in order to assess 
the extent of perineural invasion) (3). The main aim of the treatment for cSCC is the complete eradication of the tumor or metastases when possible (3). Therefore, the gold standard treatment for cSCC is surgery (3).

Our patient was an 83-year-old T2DM male with a positive oncological history and a chronic ulcer on his foot. Importantly, the appearance of suspected skin lesions in a T2DM patient with a positive oncological history requires much attention. An accurate evaluation of the patient is paramount in choosing the adequate therapeutic algorithm as well as the need for a multidisciplinary team. Given the unusual features of the foot lesion, the chronicity as well as the presence of calcification detected by radiography (which may be indicative of a chronic inflammatory process or a malignant degeneration), we decided to perform a histological investigation. We found an in situ cSCC with no characteristics of malignancy (Fig. 2, Panel B, C and D). Seeing that the inguinal and the popliteal cavity ultrasound did not reveal the presence of suspicious lymph nodes, we did not perform further radiological examinations.

To date, the physiopathology of cSCC is not completely understood. The cause seems to be multifactorial, including advanced age, sunlight or other ultraviolet radiations, immunosuppression, human papillomavirus, chronic scarring conditions and certain familial cancer syndromes $(1,2,3)$. In our case, several risk factors for cSCC can be identified, such as old age, male sex, presence of T2DM, prior history of a colon adenocarcinoma and a chronic infection with possible recurrent trauma (which is frequently exacerbated by ill-fitting footwear in the presence of diabetic peripheral neuropathy). Familial cancer syndromes were not actively indagated.

Diabetes mellitus is strongly associated with insulin resistance and chronic inflammatory state, contributing to the local and systemic release of reactive oxygen species and several mediators, including interleukin-1 (IL-1), IL-6 and tumor necrosis factor-alpha (TNF-alpha) and tumor growth factor-beta (TGF-beta) (6). In addition, presently, there are data to indicate that all these (diabetes-related) mediators can promote development and progression of some cancers by cellular growth and proliferation, angiogenesis, inhibition of cellular apoptosis and DNA damage (6). Experimental studies also suggest that, compared to those without T2DM, patients with T2DM have a higher level of genomic instability and epigenetic alterations, that may concur to the development of cancer (6).

Similarly, repeated trauma or chronic infection is able to generate local inflammation with the release of several molecules, such as cytokines, prostaglandins and
TGF-beta. The chronic inflammation may produce genetic and epigenetic changes, damaging DNA, inhibiting its repair, altering transcription factors, preventing apoptosis and stimulating angiogenesis, consequently concurring to the cancer formation $(11,12,13)$. In addition, a positive oncological history (as in the case of our patient) may suggest the presence of a genome highly susceptible to develop multiple cancerous mutations. For instance, several studies have reported that patients with colorectal cancer may have important molecular alterations predisposing to genetic instability, such as chromosomal instability, deficient DNA mismatch repair and epigenetic silencing of key genes (14).

Notably, all these factors should guide the clinicians to make an appropriate and accurate assessment of a lesion if it does not heal, becoming chronic. In fact, timely surgical resection of a chronic, non-healing diabetic foot ulcer might ideally preclude the development of a cutaneous squamous cell carcinoma. Therefore, when a lesion does not heal within 8-12 months (after excluding the presence of other important concomitant conditions, including peripheral arteriopathy), a biopsy should be performed to exclude the presence of a neoplasm.

In conclusion, our case report corroborates and expands previous observations $(7,8,9)$, reinforcing the notion that cSCC may be a serious (albeit rare) complication of the diabetic foot in elderly T2DM patients. The diagnosis of cSCC should always be considered and ruled out in a chronic non-healing lesion, especially in consideration of the latest risk factors for the development and progression of cSCC, in order to prevent a procrastination in the diagnosis and treatment and to improve outcome.

Declaration of interest

The authors declare that there is no conflict of interest that could be perceived as prejudicing the impartiality of the case reported.

\section{Funding}

This research did not receive any specific grant from any funding agency in the public, commercial or not-for-profit sector.

\section{References}

1 Rogers HW, Weinstock MA, Coldiron BM \& Feldman SR. Incidence estimate of nonmelanoma skin cancer (keratinocyte carcinomas) in the U.S. population, 2012. JAMA Dermatology 2015151 1081-1086. (https://doi.org/10.1001/jamadermatol.2015.1187)

2 Muzic JG, Schmitt AR, Baum CL, Wright AC, Alniemi DT, Zubair AS, Olazagasti Lourido JM, Sosa Seda IM \& Weaver AL. Incidence and trends of basal cell carcinoma and cutaneous squamous cell 
Endocrinology

Diabetes \& Metabolism

CASE REPORTS
A Mantovani and others

Cutaneous squamous carcinoma and diabetic foot
ID: 18-0065; July 2018 DOI: $10.1530 / E D M-18-0065$ carcinoma: a population-based study in Olmsted County, Minnesota, 2000 to 2010. Mayo Clinic Proceedings 201792 890-898. (https://doi. org/10.1016/j.mayocp.2017.02.015)

3 Motley R, Kersey P \& Lawrence C. British association of dermatologists; british association of plastic surgeons; royal college of radiologists, faculty of clinical oncology. Multiprofessional guidelines for the management of the patient with primary cutaneous squamous cell carcinoma; british association of dermatologists. British Journal of Dermatology 2002146 18-25. (https://doi.org/10.1046/j.0007-0963.2001.04615.x)

4 Massari LP, Kastelan M \& Gruber F. Epidermal malignant tumors: pathogenesis, influence of UV light and apoptosis. Collegium Antropologicum 200731 83-85.

5 Guariguata L, Whiting DR, Hambleton I, Beagley J, Linnenkamp U \& Shaw JE. Global estimates of diabetes prevalence for 2013 and projections for 2035. Diabetes Research and Clinical Practice 2014103 137-149. (https://doi.org/10.1016/j.diabres.2013.11.002)

6 Klil-Drori AJ, Azoulay L \& Pollak MN. Cancer, obesity, diabetes, and antidiabetic drugs: is the fog clearing? Nature Reviews Clinical Oncology 201714 85-99. (https://doi.org/10.1038/nrclinonc.2016.120)

7 Marie-France K, Rajesh J, Vidhu N, Rachel B \& Stephen J. Squamous cell carcinoma in a heel ulcer in a patient with diabetes. Diabetes Care 200831 e57. (https://doi.org/10.2337/dc08-0284)

8 Chiao H-Y, Chang S-C, Wang C-H, Tzeng Y-S \& Chen S-G. Squamous cell carcinoma arising in a diabetic foot ulcer. Diabetes Research and Clinical Practice 2014104 e54-e56. (https://doi.org/10.1016/j. diabres.2013.12.027)

9 Larijani B, Tavangar SM \& Bandarian F. Squamous cell carcinoma arising in a chronic, nonhealing diabetic foot ulcer. Wounds 201729 E48-E50.

10 Quitkin HM, Rosenwasser MP \& Strauch RJ. The efficacy of silver nitrate cauterization for pyogenic granuloma of the hand. Journal of Hand Surgery-American 200328 435-438. (https://doi.org/10.1053/ jhsu.2003.50092)

11 Kawanishi S, Hiraku Y, Pinlaor S \& Ma N. Oxidative and nitrative DNA damage in animals and patients with inflammatory diseases in relation to inflammation-related carcinogenesis. Biological Chemistry 2006387 365-372. (https://doi.org/10.1515/ BC.2006.049)

12 Kundu JK \& Surh YJ. Inflammation: gearing the journey to cancer. Mutation Research 2008659 15-30. (https://doi.org/10.1016/j. mrrev.2008.03.002)

13 Keibel A, Singh V \& Sharma MC. Inflammation, microenvironment, and the immune system in cancer progression. Current Pharmaceutical Design 200915 1949-1955. (https://doi. org/10.2174/138161209788453167)

14 Kawakami H, Zaanan A \& Sinicrope FA. Implications of mismatch repair-deficient status on management of early stage colorectal cancer. Journal of Gastrointestinal Oncology 20156 676-684. (https:// doi.org/10.3978/j.issn.2078-6891.2015.0650

Received in final form 21 June 2018 Accepted 9 July 2018 